\section{A. Winter}

Institute for Medical Informatics, Statistics and Epidemiology

University of Leipzig

Leipzig, Germany

\title{
Synopsis
}

\section{Health Information Systems}

- in the right place

- for health care professionals, public health authorities and for the patient

- in the right form

- tohelp professionals, authorities and patients to make the right decisions.

Making the right decision requires selecting both the relevant patient related information and the matching medical knowledge, which has to be combined in the end. The act of combining information and knowledge, i.e. the decision making, should be up to the professional and the patient. But in an age of explosively increasing medical knowledge, the process of selecting knowledge and information should be supported by components of the HealthIS.

The first paper by DouPI and VAN DER LEI addresses this task of HealthIS. They combine patient related information and medical knowledge, but they donot do it for physicians or authorities, but for the patients to support their education and their empowerment.

The paper confirms the importance of having patient record data in a structured or coded way. Since it is still a challenging task to derive that data from freetext, DouPI and VAN DER LEI's approach of structuring all data at the time of data entry is promising. Rightly they argue that providing tailored information for patients using medical record data is an additional benefit of routine medical record documentation. On the one hand, this means that we can be thankful for all the worldwide efforts of introducing diagnosis related groups (DRG), which enforce health providers to encode at least their diagnoses and procedures properly. And on the other hand applications like this are very important to motivate doctors to do the annoying and time consuming job of documenting and coding very carefully.

Motivating doctors to use computer based tools for their documentation and managing tasks is not so easy, especially if only a single activity but not the entire (business) process is considered. In those cases one can find that e.g. physician order entry can require substantially more time than traditional paper-based methods [1]. Benefits for staff and for patients can therefore only be achieved if the entire process is taken into account (as e.g. in the fourth paper of this section) or if the multiple usability of electronic data is exploited [2].

The latter has been done by LAZARUS et al. in the third paper. They used a health information system as a real regional information system, and contribute to peoples' health by supporting public health authorities in recognizing problems. The authors developed a surveillance system which gathers data from ambulatory encounters to detect 
acuteillness clusters, including potential bioterrorism events in the region. The data is presented to public health personnel in a form that enables efficient decision making. Such a surveillance system needs the interconnection of application components of the health information system and the possibility to collect data from these components at least temporarily in a central database for analysis. A simple fact - but very often forgotten. The authors could make use of a comfortable situation because the data needed was already stored in one central database. It is the database of one electronic patient record system used by a large multispecialty group practice, serving 250.000 people, i.e. about $10 \%$ of the region's population. The authors' surveillance system exploits the timely, valid, and inexpensive data, especially about diagnoses and addresses of patients, which is still documented for every ambulatory encounter within this electronic patient record system. So there has been no need to collect data from different sources. The diagnoses are grouped into rough clusters. Statistical methods are used to detect significant deviations concerning the number of events within the clusters during the last 24 hours compared to the numbers in the past. Regional health care authorities receive a valuable means for detecting public health problems, which in addition can be adjusted to special interests and individual thresholds.

Basically the term "health information system" is a promise. Being sceptical the term may even be considered as being mealy-mouthed or "newspeak" as in the novel "1984" by George Orwell.

Do these information systems really contribute to the health of people or do they - if at all - contribute only to cost savings at the supported institutions?

If we as medical informatics professionals realize our professional responsibility for the objects of our work (as laid down e.g. in [3]), it becomes clear that the critical reflection of this question is an ethical duty to fulfill everyday in our professional life.

The second and fourth paper of this section show the methodological background to deal with this problem and give answers to the question above. But these papers also demonstrate that evaluation is a hard job.

Fraenkel et al. report on the introduction of an application for documentation and patient data management in intensive care units (ICU). They could verify significant reductions in the rates of medication, intravenous therapy, and ventilator incidents. It is intriguing, that during a brief period of return to paper-based prescribing an increased number of adverse medication events could be seen - and could be reduced again after return to computer-based prescribing. The study confirms as well, that not only the very high workload of documentation tasks in an ICU can be reduced by an appropriate computer support, but that it can also lead to a higher degree of contentment among nursing staff. In 'normal' wards or ambulatory settings, if the workload for medical and nursing documentation may not be as high as at an ICU, it may be harder to validate time savings by using computer based tools for documentation [4].

The fourth paper by MEKHJIAN et al. deals with the validation of beneficial effects by using physician order entry systems. The objective of their study was to quantify and document immediate tangible benefits on patient care. Reductions could be demonstrated in the time between the order and administration of the medication, the time for completion of radiology procedures, and the time for reporting the results of laboratory tests. Despite the improvement it was not possible to demonstrate a consistent effect on length of stay or cost. An immediate effect to patients' health could be identified considering the entire process of medication administration. A homogenous computer support for this process yields a complete reduction of transcription errors. This contributes not only to a reduction of some trouble at the ward and pharmacy but reduces harm to the patients. In their discussion the authors make clear that "because of a number of limitations, the benefits outlined ... may not be generalizable to other institutions". Readers should have this warning in mind when interpreting any other evaluation study.

To sum it up it can be said that this section presents a very encouraging sample of papers. They show us that it is possible both to design HealthIS as being beneficial for patients and to prove that they are doing so.

\section{References}

1. Overhage JM, Perkins S, Tierney WM, McDonald CJ. Controlled trial of direct physician order entry: effects on physicians' time utilization in ambulatory primary care internal medicine practices. $\mathbf{J}$ Am Med Inform Assoc 2001;8(4):361-71.

2. Haux R. Knowledge-based decision support for diagnosis and therapy: on the multiple usability of patient data. Methods Inf Med 1989;28(2):69-77.

3. IMIA Working Group 04 Data Protection in Health Information Systems. Code of Ethics for Health Information Professionals. http://www.imia.org/pubdocs/ Code_of_ethics.pdf, 2002.

4. Bürkle T, Kuch R, Prokosch H-U, Dudeck J. Stepwise Evaluation of Information Systems in a University Hospital. Methods Inf Med 1999;38:9-15.

Address of the author:

Alfred Winter

Institute for Medical Informatics, Statistics and Epidemiology

University of Leipzig

Liebigstraße 27

D-04103 Leipzig

Germany

Tel: $\quad$ +493419716107

Fax: $\quad$ +493419716109

E-mail: alfred.winter@imise.unileipzig.de

http://people.imise.uni-leipzig.de/ alfred.winter/ 